\title{
Current trends in virtual colonoscopy
}

Zarina I Lockhat, FFRad(D)SA

Department of Radiology, Pretoria Academic Hospital and University of Pretoria

Irma van de Werke, FRCR

Department of Radiology, Kalafong Hospital and University of Pretoria

André du Plessis, MB ChB

Department of Radiology, Pretoria Academic Hospital and University of Pretoria

\section{Introduction}

Conventional colonoscopy is the current gold standard procedure to evaluate the colon, as it examines the whole colon and allows for biopsy of lesions and has therapeutic potential (polypectomy). However it is regarded as an invasive procedure and has potentially serious complications.

Computed tomography (CT) colonography may have a unique role in colorectal cancer screening. The main advantages are the ability to observe the whole colon quickly without sedation or with minimal risk of perforation. It could replace barium enema as the conventional radiological investigation of the colon. Concerns about poor sensitivity and inter-observer variation have limited its use by the gastro-enterological community. ${ }^{1}$

\section{Indications for CT colonoscopy ${ }^{1}$}

CT colonoscopy is indicated in the following cases: $(i)$ as a complementary tool for imaging the colon; (ii) in preference to barium enema for incomplete colonoscopy; (iii) in the frail and elderly to exclude colorectal cancer rather than to detect polyps; and (iv) if the patient is unable or unwilling to undergo colonoscopy. ${ }^{1}$

In December 2003 Pickhardt and colleagues ${ }^{2}$ published a landmark study in CT colonography, using a cohort of 1233 asymptomatic individuals undergoing colorectal cancer screening. They showed that CT colonography not only matched conventional colonoscopy for detection of significantly sized adenomas, but outperformed it for lesions $8 \mathrm{~mm}$ or larger. ${ }^{1}$

\section{Procedure}

\section{Patient preparation}

The purpose of bowel preparation is to clean out the colon before imaging. In our unit the patient is prepared as for conventional colonoscopy. Preparations can be the so-called wet preparations, such as polyethylene glycol and sodium phosphate preparations or the drier laxatives including magnesium citrate preparations, fleet enemas, bisacodyl tablets and LoSo Prep. Generally phospho-soda (fleet enema) is recommended for young and healthy patients while the polyethylene glycol preparation is preferable for the elderly.

Prepless techniques are currently also being investigated, requiring faecal tagging methods. This is achieved by the use of orally ingested agents, usually dilute barium or iodinated contrast medium that 'tag' or 'label' residual fluid or faecal matter.

A single dose of laxative together with three doses of $250 \mathrm{ml} 2.1 \% \mathrm{w} / \mathrm{v}$ barium sulphate the day before the scan may equal diagnostic performance in fully prepared patients. ${ }^{3}$ Image analysis requires a dedicated CT colonographic software package to 'subtract' the high attenuation labelled faecal residue from the colonic lumen, i.e. CT colonographic software package with a subtraction capability.

\section{Data acquisition}

Thin-slice acquisition protocols with multidetractor row $\mathrm{CT}$ to cover the entire abdomen in a single breath hold should be used; $2.5 \mathrm{~mm}$ or $1.25 \mathrm{~mm}$ collimation is recommended.

\section{Positioning}

Scanning usually begins in the supine position and is subsequently performed in the prone position if fluid is present in the colon. The second acquisition is to ensure that fluid-filled segments can be interpreted later.

\section{Premedication}

The efficacy of administering Buscopan or glucagon before scanning to improve colonic distension is controversial. In a study of 240 patients who underwent virtual colonoscopy, Rogalla and colleagues ${ }^{2}$ found that glucagon improved distension significantly only when the results were analysed per segment; however 
Buscopan provided better volume distension and significantly reduced the number of collapsed colonic segments.

\section{Insufflation}

Automated insufflation with carbon dioxide $\left(\mathrm{CO}_{2}\right)$ using an automatic insufflation device is recommended as this maintains a constant $\mathrm{CO}_{2}$ pressure during scanning. Where an automatic insufflator is not available, a hand pump may be used with insufflation of room air.

\section{Low-dose CT}

Intrinsic high contrast between the colonic wall and insufflated gas allows dose-saving low MA protocols (e.g. $50 \mathrm{mAs}$ ). Recent data suggest that excellent sensitivity for cancer and polyps of over $6 \mathrm{~mm}$ can be achieved using a collimation of $2.5 \mathrm{~mm}$ and tube current of $10 \mathrm{mAs}$ giving an effective dose of $2.15 \mathrm{mSV}$ in men and $2.75 \mathrm{mSV}$ in women. ${ }^{1}$

\section{Intravenous contrast for problem solving}

The use of contrast is also controversial in virtual colonoscopy. A study performed by Martina Morrin and colleagues ${ }^{2}$ found that sensitivity improved from $58 \%$ to $75 \%$ with the use of IV contrast. Intravenous contrast is helpful if there is poor preparation of the patient and should be used as a problem-solving tool. ${ }^{2}$

\section{Interpretation of data}

Interpretation of data can be performed by a 3D flythrough endoluminal approach, with simultaneous correlation with 2D axial images and 2D MPR images, software packages which include multiple imaging layout formats for adequate visualisation of the entire colon.

As CT colonography becomes more widespread, there is increasing inter-observer variation in interpretation. Computer-aided diagnosis (CAD) plays an important role in this regard. When applied to the colon, CAD relies on three main steps: (i) extraction of the colon from the 3D CT volume; (ii) identification of potential polyp candidates; and (iii) eliminating false positives as far as possible.

\section{Detection of lesions}

In a study performed by Pickhardt et al. ${ }^{2}$ polyps greater than $6 \mathrm{~mm}$ were detectable using $1.25-2.5 \mathrm{~mm}$ collimation with multidetector row CT. Sensitivity and specificity of CT colonography decreases as lesion size decreases below $5 \mathrm{~mm}$.
The gastroenterological literature emphasises the advanced adenoma as an appropriate target for screening. The advanced adenoma is classified by size or histology, with lesions greater than $10 \mathrm{~mm}$ or with villous histology being significant.

There has been debate about significance of small lesions in CT colonography screening or surveillance programmes; however practically this is not an issue for endoscopic screening techniques, as all lesions seen are removed. However with radiological screening, Pickhardt et al. ${ }^{2}$ have considered a cut-off size of $8 \mathrm{~mm}$ or greater as a recommendation for conventional colonoscopy, and recommend repeat follow-up scans for smaller lesions after $2-3$ years.

Figures $1-8$ illustrate the procedure.

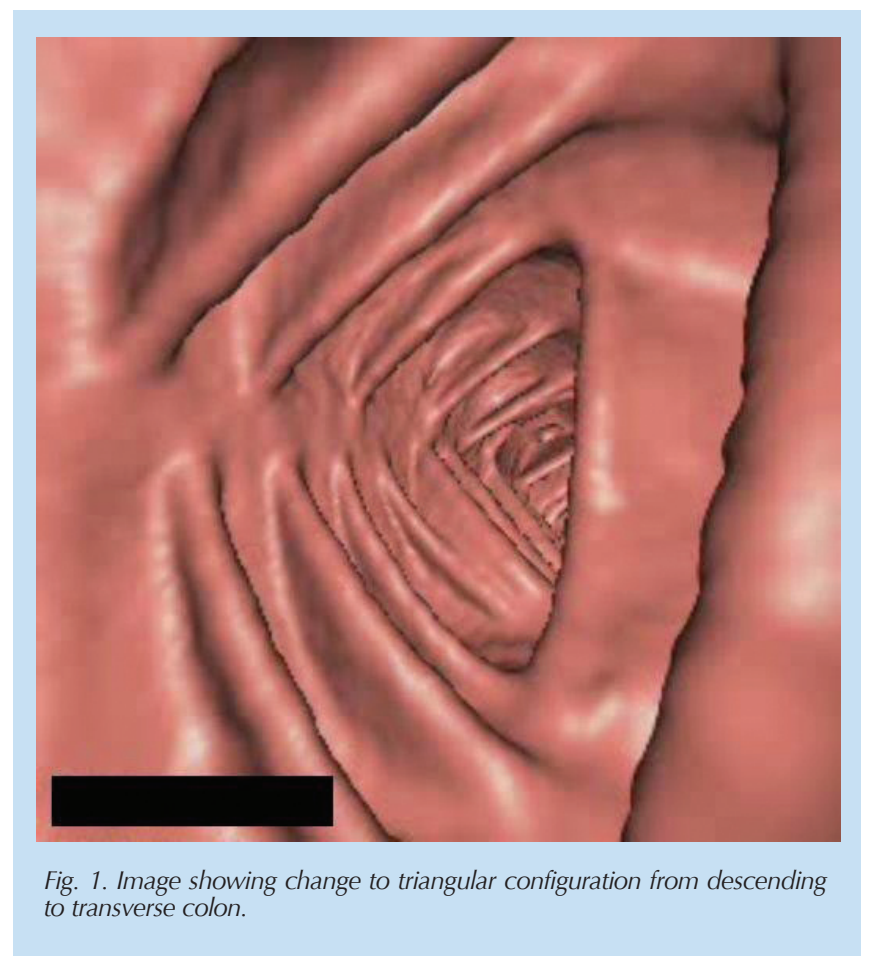

\section{Incidental extra-colonic findings}

A study performed by Gluecker and colleagues ${ }^{7}$ reported that up to $60 \%$ of patients had an extra-colonic abnormality that required further investigation, and $10 \%$ of these were deemed of high clinical importance. The low-dose radiation protocols used in virtual colonoscopy provide adequate detail of the colon, but limited views of extra-colonic organs, and thus provide restricted interpretation of these structures. 


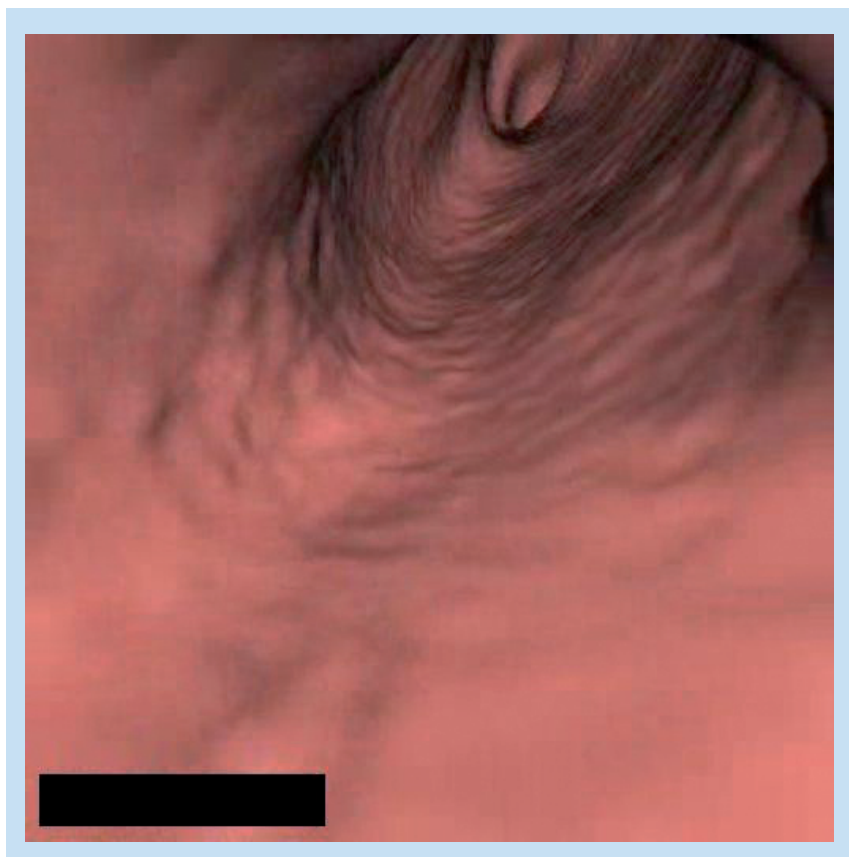

Fig. 2. Example of irregular mucosa.

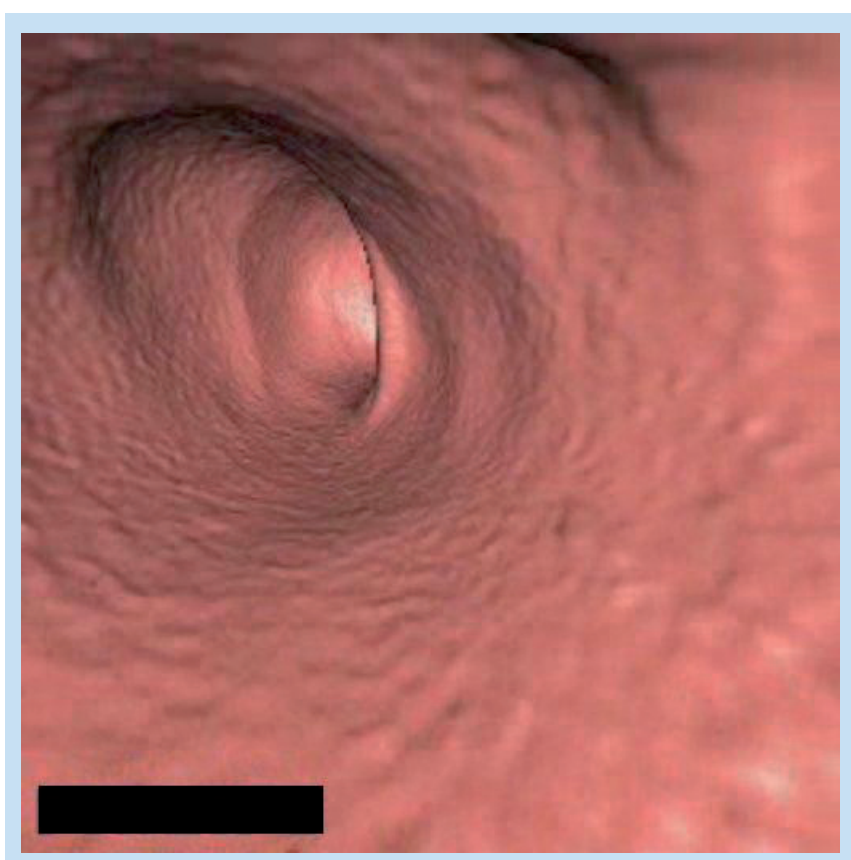

Fig. 3. Example of irregular mucosa.

\section{Current development in magnetic resonance colonography}

Magnetic resonance colonography (MRC) is also emerging as a potential complementary investigation for the diagnosis of colorectal cancer and the benign pathol-

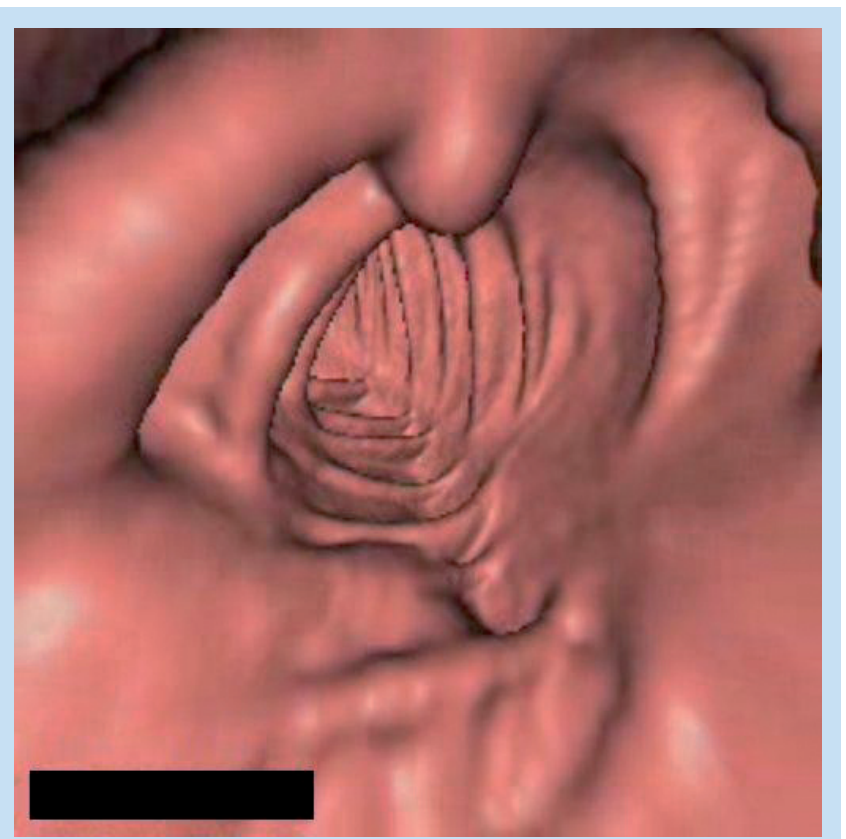

Fig. 4. Example of a polyp.

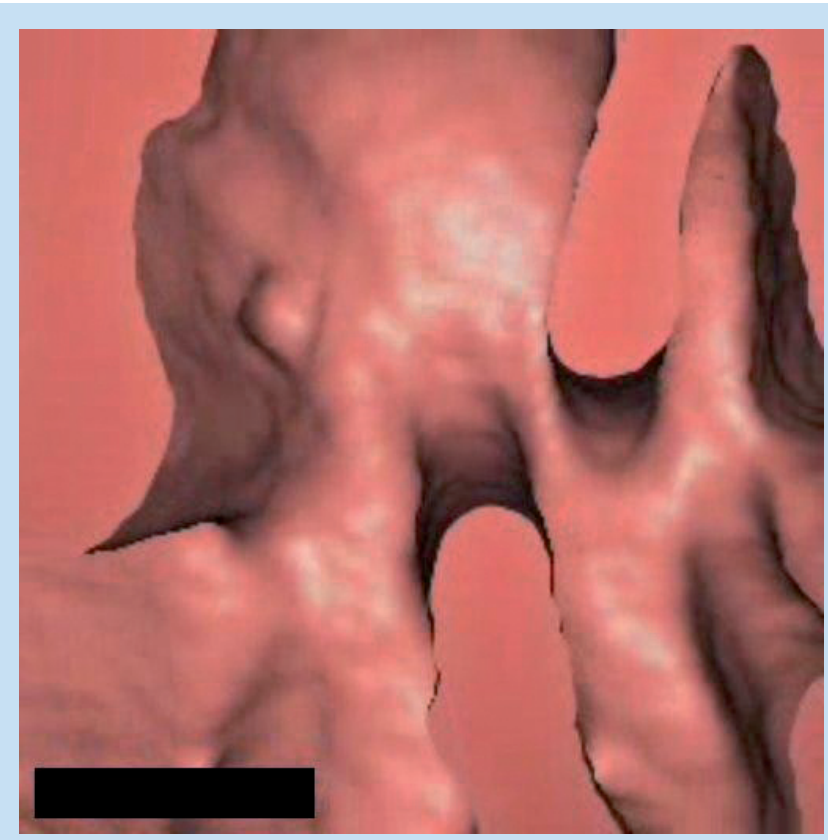

Fig. 5. Example of a small polyp.

ogy. MRC is based on the acquisition of datasets of consecutive images which are subsequently segmented and rendered so as to produce reconstructed $2 \mathrm{D}$ and $3 \mathrm{D}$ images. MRC relies on ultra-fast, T1-weighted 3D gradient-echo data acquisitions collected during a single breath hold. For MRC, the colon is filled with water combined with a paramagnetic contrast agent such as 


\section{REVIEW ARTICLE}

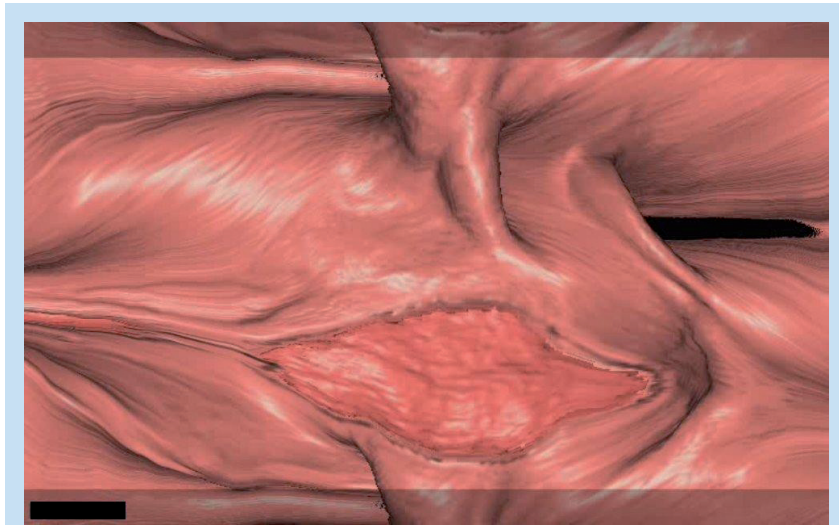

Fig. 6. Pseudo-ulcer appearance due to free fluid in the bowel.

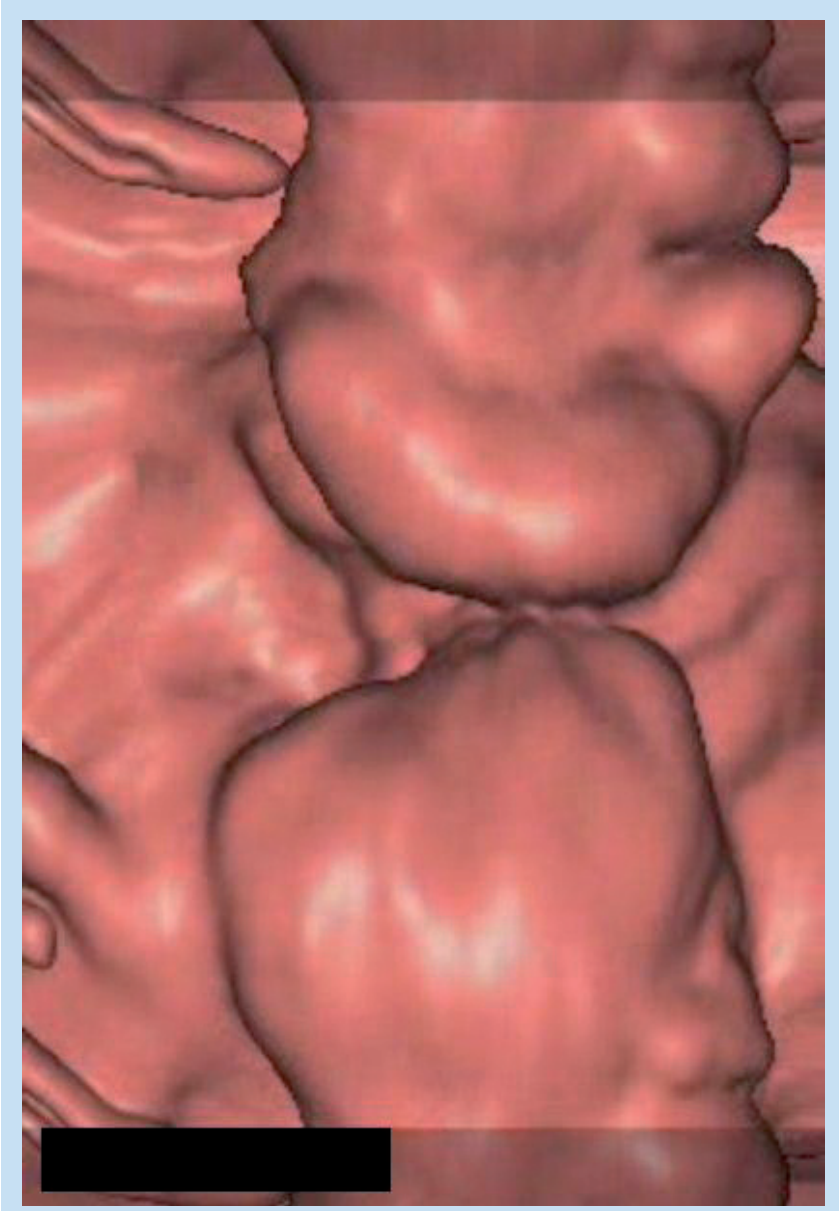

Fig.7. Example of carcinoma of the rectum demonstrating irregular mass with narrowing of the lumen.

gadolinium. The lumen of the colon therefor appears bright and the walls dark. Lesions within the wall protrude into the bright lumen, appearing as filling defects. The difficulty in diffentiating masses from faeces can be by avoided by imaging the patient prone and supine, and

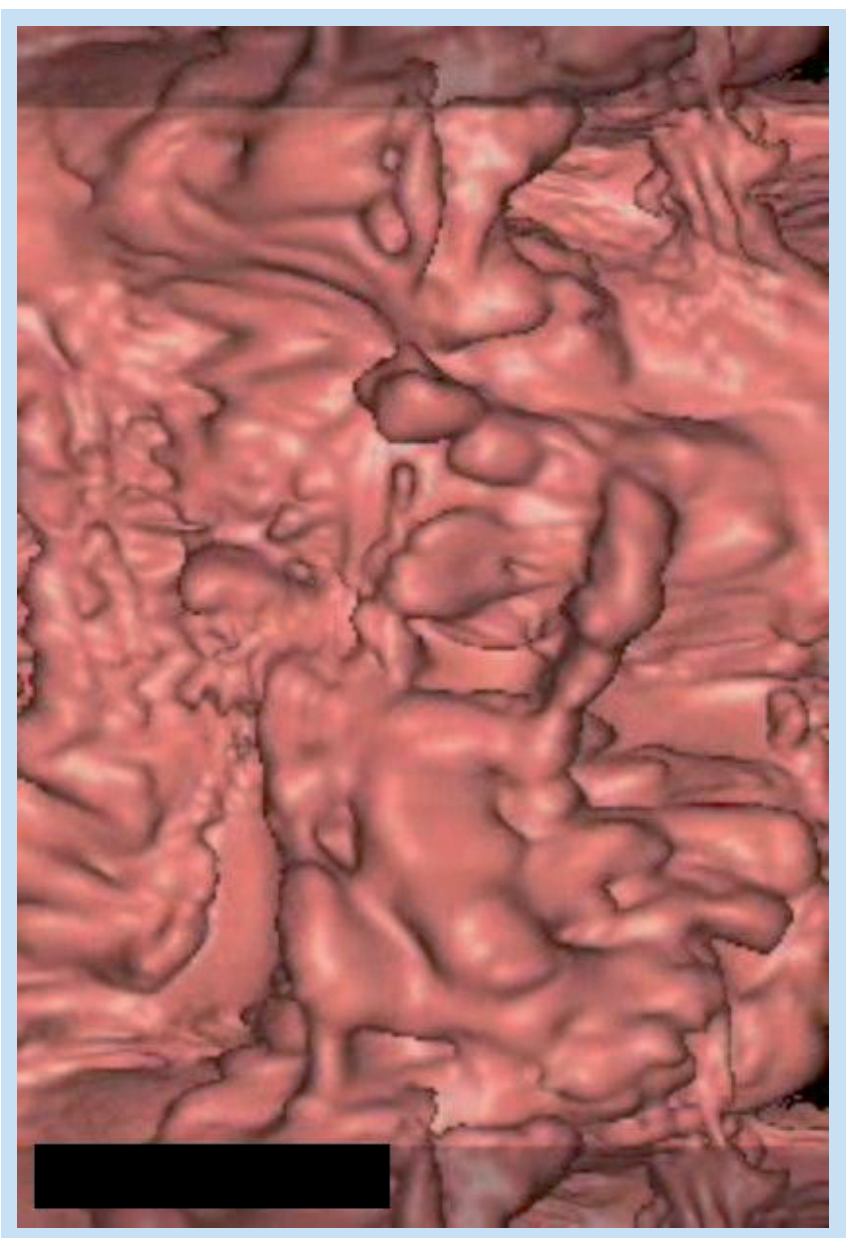

Fig. 8. Appearance due to excessive residual faecal matter.

more recently by using techniques to render the lumen and faeces dark while enhancing the colonic walls. This faecal tagging, or dark-lumen colonography method, is facilitated by the administration of barium sulphate orally and as an enema, which then renders the lumen and faeces dark. Subsequent intravenous administration of gadolinium causes the colonic wall to appear bright. ${ }^{3}$ This is further aided by applying a fly-through of the colon to double-check for small lesions.

As with new technology, the diagnostic accuracy of MRC is expected to improve. This highlights the need for further evaluation of the role of MRC, CTC and CC.

\section{Conclusion}

Virtual colonoscopy continues to evolve. CT colonography allows non-invasive, rapid, high resolution imaging of the colon with advances in low-dose radiation, protocols, tagging techniques, $\mathrm{CAD}$ and improved detection 


\section{REVIEW ARTICLE}

of small polyps and flat adenomas. Studies have shown that when the virtual examination is performed correctly, its accuracy rivals that of conventional colonoscopy.

MR colonoscopy is currently being evaluated as a further imaging procedure, however the exact diagnostic role of MRC needs to be clarified and further evaluation is necessary to refine its application and diagnostic accuracy in comparison with CT colonography, conventional colonoscopy and other imaging methods.
REFERENCES

1. Nicholson F, Talor S, Halligan S, Kamin M. Recent developments in CT colonography. Clin Radiol 2005; 60: $1-7$.

2. Barnes E. VC acquisition: Simple steps optimise imaging results. Virtual colonoscopy digital community. Aunt Minnie.com staff writer. 24/8/2005.

3. Purkayastha S, Tekkis P, Athanasiou T, et al. Magnetic resonance colonography versus colonoscopy as a diagnostic investigation for colorectal cancer: a meta-analysis. Clin Radiol 2005; 60: 980-989.

4. Barnes E. Best preps are tailored to VC reading method. Virtual colonoscopy digital community. Aunt Minnie.com staff writer. 16/11/2005.

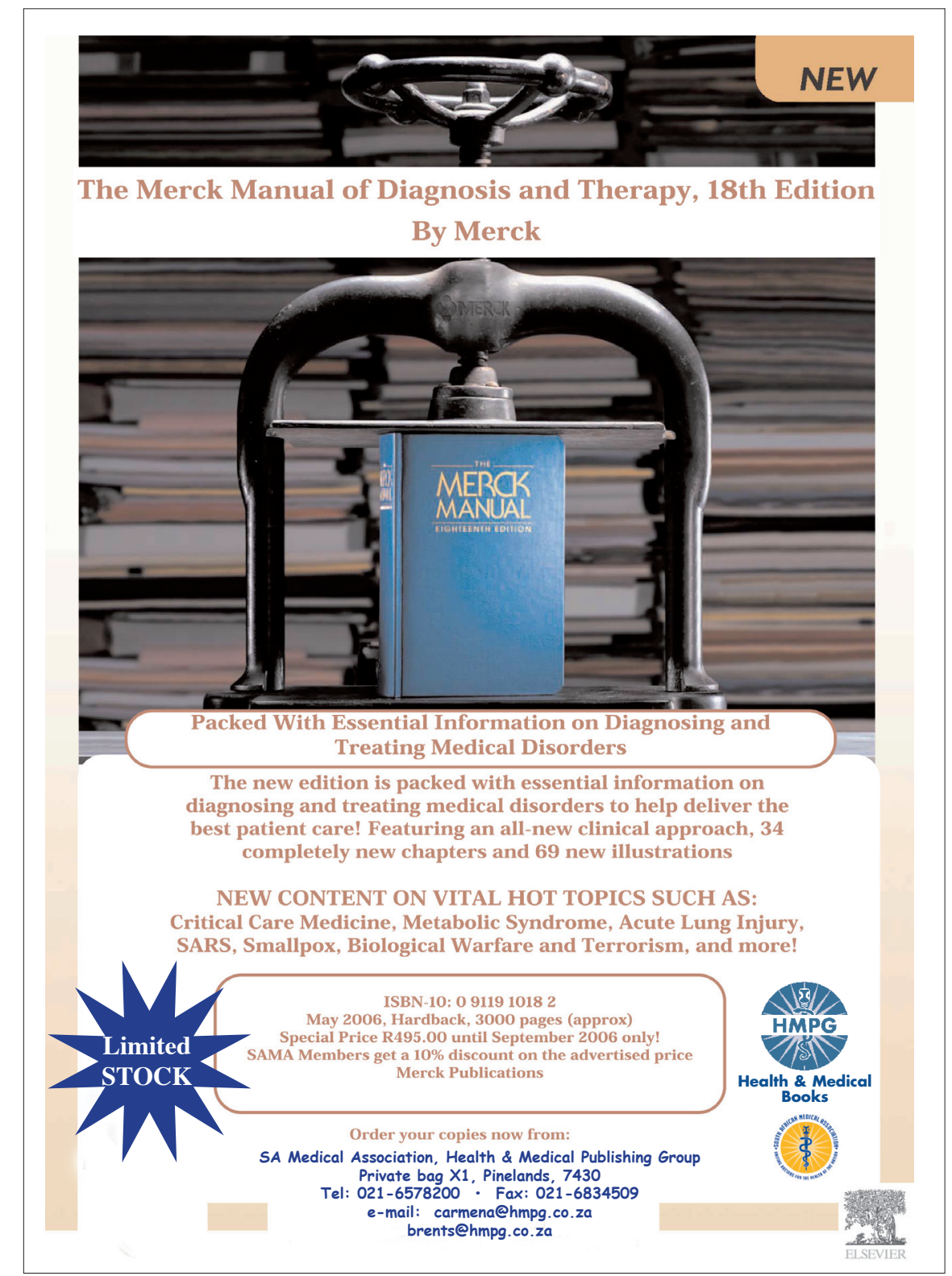

